\title{
Efecto de la bajada de peso y la competición sobre el perfil de estado de ánimo en deportes de combate
}

\section{Weight cutting and competition effects on mood state profile on combat sports}

\author{
Efeito de menor peso e concorrência no perfil \\ de humor em esportes de combate
}

\author{
Alberto Martínez-Abellán, Ricardo Morán-Navarro, José María López-Gullón, Jesús G. Pallarés, Ernesto de la Cruz- \\ Sánchez y Francisco Ortín
}

\begin{abstract}
Resumen: El propósito de este estudio ha sido describir los cambios que sufre el estado de ánimo que presentan los deportistas de combate durante las fases de bajada de peso y su posterior recuperación antes del comienzo de los eventos competitivos prioritarios de la temporada. Un total de 105 hombres y mujeres especialistas en las modalidades de combate de lucha olímpica, taekwondo y boxeo fueron pesados y contestaron la versión abreviada del Perfil de Estados de Ánimo (POMS) entre 30 y 60 minutos antes del pesaje oficial (PRE) y entre 30 y 60 minutos antes del comienzo del primer combate (POST) de sus respectivos campeonatos nacionales. Los resultados demostraron que las hubo mejoras significativas $(\mathrm{p}<.05)$ en las dimensiones de Cólera, Fatiga y Depresión, aunque estos cambios no parecen estar relacionados con la variación porcentual de la masa corporal que experimentan los deportistas durante este mismo periodo $(r<.261)$. Estos hallazgos nos indican que las 12-18 horas que separan el pesaje oficial y el comienzo del torneo en deportes de combate propician mejoras sustanciales en sus estados de ánimo, aunque estos cambios parecen ser independientes de sus fluctuación de la masa corporal.

Palabras clave: Estados de ánimo, deportes de combate, pérdida de peso; lucha, taekwondo, boxeo.

Abstract: The purpose of this study was to describe changes in the mood state of combat sport athletes during the weight cutting phases and subsequent recovery before the start of the main competitive events of the season. One hundred and five men and women combat sport specialist of Olympic wrestling, taekwondo and boxing were weighted and answered the abbreviated version of the Profile of Mood States (POMS) 30 to 60 minutes before the official weigh-in (PRE) and 30 to 60 minutes before the start of the first bout (POST) of their respective national championships. The results
\end{abstract}

showed significant improvements $(\mathrm{p}<.05)$ in the Anger, Fatigue and Depression dimensions, although these changes do not appear to be related to the percentage change in the body mass experienced by these athletes during the same period $(r<.261)$. These findings indicate that the 12-18 hours that separate the official weigh-in and the start of the tournament produce substantial improvements on the athletes' mood state, although these changes seems to be independent on their body mass oscillations.

Key-words: mood states, combat sports, weight loss, Olympic wrestling, taekwondo, boxing.

Resumo: O objetivo deste estudo foi descrever as alteraçôes sofridas pelo clima que os atletas lutaram durante as fases de redução de peso e subsequente recuperação antes do início dos eventos prioritários da temporada competitivos. Um total de 105 homens e mulheres especialistas nas categorias de combate de wrestling, taekwondo e boxe foram pesados e responderam a versão curta do Profile of Mood States (POMS) entre 30 e 60 minutos antes da pesagem oficial (PRE) e entre 30 e 60 minutos antes do início do primeiro ataque (POST) nos seus respectivos campeonatos nacionais. Os resultados mostraram que houve melhora significativa $(\mathrm{p}<0,05)$ nas dimensōes de raiva, fadiga e depressão, embora estas alteraçôes não parecem relacionadas com a variaçáo percentual de massa corporal vivida por atletas durante este mesmo período $(\mathrm{r}<0,261)$. Estes resultados indicam que as 12-18 horas que separam a pesagem oficial eo início do torneio em combate esportes adotivos melhorias substanciais em seus humores, mas essas mudanças parecem ser independentes de sua flutuação de massa corporal. Palavras-chave: humores, esportes de combate, perda de peso; wrestling, taekwondo, boxe.

\section{Introducción}

La inmensa mayoría de los deportes de combate se rigen por un sistema de categorías por pesos que trata de equilibrar el potencial físico entre rivales, y de esta forma aumentar el porcentaje del rendimiento que depende de las habilidades técnico-tácticas y psicológicas que demuestre cada atleta durante el combate (Burke y Cox, 2009; Chiodo et al., 2011;

Dirección para correspondencia [Correspondence address]: Jesús G. Pallarés. E-mail: jgpallares@um.es
García-Pallarés, López-Gullón, Muriel, Díaz e Izquierdo 2011; García-Pallarés, López-Gullón, Torres-Bonete e Izquierdo, 2012; Hernández, Torres-Luque y Olmedilla, 2009; Langan-Evans, Close y Morton, 2011; Martínez-Abellán, 2013). Como principal consecuencia de esta característica del reglamento, la masa corporal de estos deportistas se verifica unas pocas horas antes del comienzo de cada torneo en el denominado "pesaje oficial", a partir del cual se establecen los diferentes cuadros de competición en cada una de las ca- 
tegorías de peso. Por ello, durante las fases de puesta punto de los principales eventos competitivos de la temporada (2-6 semanas previas), la mayoría de los deportistas de combate reducen su masa corporal para tratar de alcanzar una categoría de peso inferior donde obtener alguna ventaja física frente a sus rivales (Horswill, 1992). Esta aproximación a la competición, conocida internacionalmente como "Weight Cutting" y sus consecuencias sobre el propio rendimiento específico, fisiológico, neuromuscular y cognitivo han sido ampliamente estudiadas en la literatura (Hernández et al., 2009; Horswill, 1992; Marttinen, Judelson, Wiersma y Coburn, 2011).

Para estas disciplinas de combate, las pérdidas de peso están incluidas en su filosofía como deporte y son ejecutadas por la mayoría de atletas de forma tradicional, sin plantearse la peligrosidad que pueden causar hacia su salud (Horswill, 1989). Además, varios estudios han demostrado que las bajadas de peso realizadas por estos deportistas, en ocasiones de forma extrema, pueden tener efectos en el rendimiento deportivo, ya sea desde el punto de vista fisiológico (Horswill, 1992) neuromuscular (Martínez-Abellán, 2013) o psicológico (Hernández et al., 2009; Marttinen et al., 2011; Torres-Luque, Hernández, Olmedilla, Ortega y Garatachea, 2013). En relación a los aspectos psicológicos, un grupo numeroso de estudios han demostrado que la rápida pérdida de peso ocasiona alteraciones en el Vigor, la Concentración, la Autoestima o en la disminución de la memoria a corto plazo, así como también, el aumento de la Confusión, la Ira, la Fatiga, la Depresión y el Aislamiento (Degoutte, Jouanel, Begue, Colombier, Lac, Pequignot y Filaire, 2006; Filaire, Maso, Degoutte, Jouanel y Lac, 2001; Hernández et al., 2009; Steen y Brownell, 1990; Torres-Luque et al., 2013; Umeda, Nakaji, Shimoyama, Yamamoto, Totsuka y Sugawara, 2004). La literatura científica señala la íntima relación que existe entre el estado de ánimo y el rendimiento deportivo, de manera que las percepciones que los atletas tienen sobre sus estados anímicos pueden influir directamente sobre diferentes habilidades técnico-tácticas (Lazarus, 2000; Ruiz y Hanin, 2004). El manejo incorrecto de los estados de ánimo puede fomentar en el atleta estrategias de afrontamiento erróneas y la aparición de niveles de activación inadecuados (De la Vega, Ruiz, Tejero y Rivera, 2014). Concretamente, un estudio reciente llevado a cabo con deportistas de combate señala que la falta de concentración afecta al competidor en torneos oficiales, pudiendo provocar una disminución de la memoria a corto plazo, rabia excesiva, autoestima baja, depresión y aislamiento (Franchini, Brito y Artioli, 2012). Todo esto puede condicionar al deportista a seguir o no las instrucciones de su entrenador antes y durante el combate, tomar decisiones anticipadas o erróneas, así como acciones ilegales, para en definitiva determinar la posibilidad de ganar o perder un enfrentamiento.

Una de las herramientas más utilizadas para recoger la información psicológica es el POMS (Profile of Mood States).
Este instrumento fue creado para el ámbito clínico, ampliando su aplicación a otros contextos como la actividad física y el deporte. El POMS fue diseñado por Mcnair, Lorr y Droppleman en 1971, con el fin de medir los efectos de la psicoterapia y la medición psicotrópica en pacientes de centros o instituciones psiquiátricas. Existen numerosos estudios que han utilizado el cuestionario POMS como instrumento para evaluar los estados de ánimo en competidores de diferentes modalidades deportivas, como atletismo (Arruza, Otaegi, González, Irazusta y Ruiz, 2011; Macedo, Sinim y Noce, 2007; Morgan y Pollock, 1977), deportes de equipo (Hoffman et al., 1999), remo (Morgan y Johnson, 1978) e incluso en deportes de combate (Hernández et al., 2009; Morgan y Johnson, 1977; Torres-Luque et al., 2013), aportando en todos ellos información muy relevante a los técnicos responsables de estos atletas para el control y manejo de sus estados de ánimo en periodos competitivos y pre-competitivos.

Por todo ello, los objetivos de este estudio fueron i) cuantificar los cambios que sufre el estado de ánimo de los deportistas de combate de alto nivel en el periodo de tiempo que separa el pesaje oficial y el comienzo del principal evento competitivo de la temporada (i.e., 12-18 horas), así como ii) estudiar la relación de estos cambios del estado de ánimo con las fluctuaciones de la masa corporal que experimentan los atletas de combate durante este lapso de tiempo.

\section{Método}

Se presenta un estudio no experimental, descriptivo longitudinal y asociativo en una muestra de deportistas de combate de tres modalidades de combate diferentes. La muestra fue seleccionada mediante muestreo incidental por el único motivo de acceso viable.

\section{Muestra}

La muestra para este estudio estuvo compuesta por un total de 105 participantes, 75 hombres (edad 22.8ะ4.1 ańos, masa corporal $74.1 \pm 15.1 \mathrm{~kg}$, talla $176.1 \pm 6.7 \mathrm{~cm}$, grasa corporal 10.0 $\pm 4.5 \%$ ) y 30 mujeres (edad $22.7 \pm 4.5$ ańos, masa corporal $57.1 \pm 8.9 \mathrm{~kg}$, talla $164.9 \pm 7.2 \mathrm{~cm}$, grasa corporal $16.6 \pm 4.4 \%$ ), especialistas de tres deportes olímpicos de combate diferentes: lucha olímpica $(\mathrm{n}=41)$, taekwondo $(\mathrm{n}=36)$ y boxeo $(\mathrm{n}=28)$ que se ofrecieron voluntariamente a participar en este estudio. Esta muestra puede considerarse perfectamente representativa de la población a estudio, no sólo por su tamaño en valor absoluto, sino porque estos 105 participantes representan el 40,1\% del total de los deportistas que compitieron en los campeonatos nacionales de la temporada 2012/2013 en estas tres modalidades de combate olímpicas, habiendo participado 35 de ellos (i.e., el 33\% del total de la muestra) en competiciones internacionales representando su respectiva federación nacional. 
Los 105 atletas tenían al menos 4 años de experiencia en el entrenamiento y competición en sus respectivas modalidades, y todos ellos alcanzaron el peso requerido por su categoría durante el pesaje oficial de su respectivo campeonato nacional en el que se realizó la fase experimental de esta investigación. Los entrenadores y deportistas fueron informados sobre los procedimientos experimentales y los posibles riesgos y beneficios del proyecto. El estudio, que cumplió con la Declaración de Helsinki, fue aprobado por la Comisión de Bioética de la Universidad de Murcia, y se obtuvo un consentimiento informado de cada deportista antes de participar.

\section{Material}

Se ha utilizado el Profile of Mood States (McNair et al., 1971) en la versión abreviada y validada al español (Fuentes, Balaguer, Meliá y García-Merita, 1995). Este cuestionario consta de 29 adjetivos, en los cuales el sujeto debe informar sobre su propio estado de ánimo durante la jornada del pesaje (PRE) y el día de la competición (POST). Cada una de estas cualidades debe establecerse con una escala numérica de 0 a 4 (siendo 0 nada, 1 un poco, 2 moderadamente, 3 bastante y 4 muchísimo). Los ítems recogen los estados anímicos de Depresión, Vigor, Cólera, Tensión y Fatiga. Con respecto al cuestionario POMS original de 58 adjetivos, esta versión reducida mostró altos niveles de validez $(\mathrm{r}=$.93) (Fuentes et al., 1995).

\section{Procedimiento}

La recogida de los datos fue realizada por psicólogos del deporte, que ayudaron en todo momento a los deportistas en la explicación y forma de rellenar el cuestionario POMS, haciendo comprender a los competidores la importancia de cumplimentar cada ítem según las sensaciones en las que se encontraban. Estos especialistas explicaron a los atletas la confidencialidad de los datos recogidos, asegurando que los resultados sólo serían utilizados con fines de investigación, para obtener una mayor implicación por parte de los sujetos.
Los participantes completaron el cuestionario en dos ocasiones: i) entre 30 y 60 minutos antes del pesaje oficial (PRE) y ii) entre 30 y 60 minutos antes del comienzo del primer combate (POST) de sus respectivos campeonatos nacionales. Igualmente, la masa corporal de todos los participantes se midió mediante una báscula calibrada (Tanita BC-418, Tanita Corp., Tokyo, Japan) durante las valoraciones PRE y POST. Este lapso de tiempo que separaba ambas medidas PRE y POST osciló entre 12-18 h, dependiendo el propio reglamento de competición de cada modalidad deportiva. Estas mediciones se realizaron en instalaciones acondicionadas a tal efecto y anexas a las que cada evento competitivo estableció para llevar a cabo el pesaje oficial de los competidores.

\section{Análisis estadístico}

Se proporcionan los estadísticos descriptivos básicos de todas las variables estudidas (media y desviación típica). La distribución de todas las variables descritas resultó no normal, según la prueba de Komogorov-Smirnov $(\mathrm{p}<.01)$, por lo que se utilizó una prueba de rangos con signo de Wilcoxon para variables relacionadas, con el objeto de describir la variación en las diferentes dimensiones analizadas durante la fase de bajada de peso. Se empleó una correlación de Spearman para analizar la relación entre el porcentaje de peso ganado y la variación de las diferentes dimensiones establecidas en el perfil de estados de ánimo. Todos los análisis fueron realizados empleando el paquete estadístico SPSS 18.0.

\section{Resultados}

El periodo de tiempo que separó el pesaje oficial (PRE) del comienzo del torneo (POST) propició un aumento significativo de la masa corporal de los participantes $(\mathrm{p}<.001)$, así como descensos significativos en las dimensiones de Cólera $(\mathrm{p}<.001)$, Fatiga $(\mathrm{p}<.001)$ y Depresión $(\mathrm{p}<.001)$, no encontrándose diferencias significativas aunque si tendencia en las dimensiones de Vigor ( $\mathrm{p}=.124)$ y Tensión $(\mathrm{p}=.146)($ Tabla 1$)$.

Tabla 1. Descriptivos básicos (media y desviación típica) y análisis del efecto de la bajada de peso (prueba de rangos con signo de Wilcoxon).

\begin{tabular}{lllllll}
\hline $\mathrm{n}=105$ & Pesaje PRE $(\mathrm{M} \pm \mathrm{DT})$ & Pesaje POST $(\mathrm{M} \pm \mathrm{DT})$ & Diferencia $(\mathrm{M} \pm \mathrm{DT})$ & Diferencia $(\%)$ & $\mathrm{Z}$ & $\mathrm{p}$ \\
\hline Peso $(\mathrm{kg})$ & $67.8 \pm 13.5$ & $68.8 \pm 13.2$ & $1.0 \pm 1.2$ & 1.5 & -6.487 & .000 \\
Vigor & $15.8 \pm 4.4$ & $16.7 \pm 4.3$ & $0.9 \pm 4.1$ & 5.7 & -1.538 & .124 \\
Cólera & $3.8 \pm 4.2$ & $2.6 \pm 3.3$ & $-1.2 \pm 2.8$ & -31.6 & -3.841 & .000 \\
Fatiga & $5.0 \pm 3.7$ & $3.0 \pm 3.0$ & $-2.0 \pm 3.2$ & -40.0 & -5.19 & .000 \\
Depresión & $2.0 \pm 3.4$ & $1.1 \pm 2.6$ & $-0.9 \pm 2.0$ & -45.0 & -3.941 & .000 \\
Tensión & $4.9 \pm 5.0$ & $5.4 \pm 4.8$ & $-0.5 \pm 3.8$ & 10.2 & -1.452 & .146 \\
\hline
\end{tabular}

La tabla 2 muestra el análisis de la relación entre el porcentaje de peso ganado PRE vs. POST) y el cambio observado en las diferentes dimensiones del perfil de estados de ánimo de la muestra estudiada. Los resultados apuntan, en líneas genera- 
les, a que el cambio en las dimensiones del perfil de estados de ánimo no guarda una relación directa con la variación porcentual de la masa corporal.

Tabla 2. Coeficiente de correlación (Rho de Spearman) entre la variación en las diferentes variables estudiadas durante la fase de recuperación post pesaje (calculadas como la diferencia entre PRE y POST; se señalan las relaciones estadísticamente significativas).

\begin{tabular}{lcccccc}
\hline & Depresión & Vigor & Cólera & Tensión & Fatiga & $\%$ Peso \\
\hline Depresión & 1.000 & -.082 & $.440^{* *}$ & .095 & $.256^{*}$ & -.190 \\
Vigor & -.082 & 1.000 & -.016 & .027 & -.132 & $-.261^{*}$ \\
Cólera & $.440^{* *}$ & -.016 & 1.000 & .159 & $.342^{* *}$ & -.016 \\
Tensión & .095 & .027 & .159 & 1.000 & .096 & -.012 \\
Fatiga & $.256^{*}$ & -.132 & $.342^{* *}$ & .096 & 1.000 & .073 \\
\% Peso & -.190 & $-.261^{*}$ & -.016 & -.012 & .073 & 1.000 \\
${ }^{*} \mathrm{p}<0.05 ;{ }^{* *} \mathrm{p}<0.001$ & & & & &
\end{tabular}

Como resultado aislado, sólo en el caso de la dimensión Vigor se observa una relación estadísticamente significativa, aunque con una correlación relativamente débil $(\mathrm{r}=-.261, \mathrm{p}=$ .014; ver figura 1).

Figura 1. Representación de la correlación entre la variación porcentual del peso ganado (\%) y el Vigor antes del pesaje (PRE) y justo antes de comenzar el torneo oficial (POST).

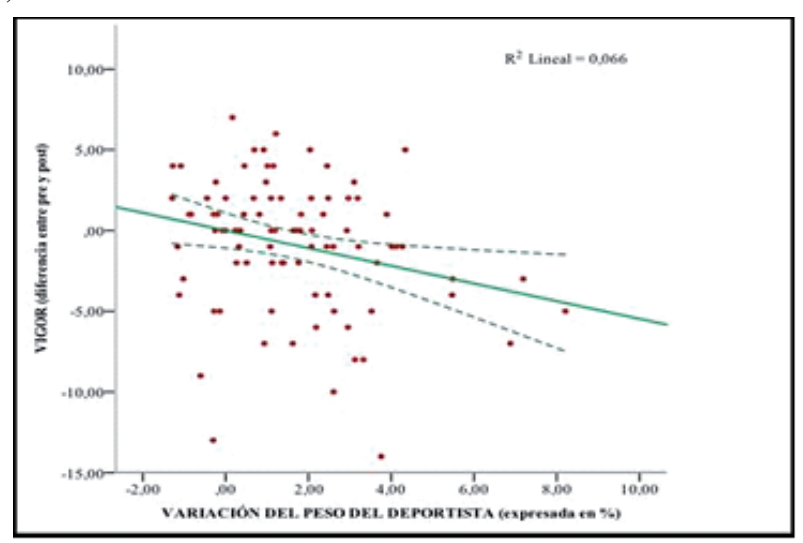

\section{Discusión}

En primer lugar, hay que destacar la importancia de las características muestrales de los deportistas, siendo todos ellos competidores de carácter nacional, incluso algunos de ellos de carácter internacional con participaciones en campeonatos europeos y mundiales. Por lo tanto, está recogida de datos y análisis posterior, pueden ejercer un importante peso en las líneas de investigación de los deportes de combate olímpicos, para ayudar a los técnicos y entrenadores en aspectos relacionados con los estados de ánimo de sus deportistas, profundi- zando y conociendo las sensaciones que pueden desarrollar los competidores el día previo a la competición e incluso el mismo día del torneo.

Los resultados de este estudio evidencian que algunas de las dimensiones de los estados de ánimo, examinados con el POMS, han sufrido mejoras considerables desde el momento del pesaje oficial hasta el comienzo del torneo. Si analizamos con más atención las cinco dimensiones del cuestionario se puede observar que existen cambios significativos en los estados de Cólera, Fatiga y Depresión con respecto al tiempo transcurrido entre las dos mediciones (PRE y POST). En cambio, no se pueden apreciar diferencias significativas con respecto al Vigor y la Tensión (ver Tabla 1).

Todos estos cambios pueden producirse ya que los participantes, el día del pesaje oficial, se encuentran cansados y exhaustos debido a las pérdidas de peso realizadas los días previos de la competición (Martínez-Abellán, 2013; Horswill, 1992). Normalmente, los deportistas que se encuentran con un peso natural situado en la mitad de dos categorías, tienden a reducir su masa corporal para competir en la categoría inferior (Franchini et al., 2012). Algunos estudios han demostrado que en los últimos siete días para una competición internacional los deportistas de modalidades de combate olímpicas pueden reducir su peso más de un 8\% (Martínez-Abellán, 2013; Horswill, 1992; Houston, Marin, Green y Thomson, 1981), e incluso pueden llegar a perder un $4 \%$ en las tres horas previas al pesaje (Smith, 1998). Por lo tanto, esto ocasiona en los competidores mucha fatiga física, pero también de carácter mental, propiciando un descenso anímico en algunas dimensiones del estado de ánimo. Estudios previos llevados a cabo con deportistas de combate han mostrado resultados similares a los descritos en este trabajo. Así por ejemplo, Hernández et al., (2009) registraron mediante el cuestionarios POMS aumentos relevantes en las dimensiones de Tensión y Vigor durante las horas previas a la competición en 10 judokas de élite. Recientemente, Torres-Luque et al., (2013), en otro estudio realizado con judokas de élite, encontraron que la carga de entrenamiento y la presencia o no de un evento competitivo afecta notablemente al estado de ánimo, destacando que el entrenamiento de carácter aeróbico implica un descenso del factor Vigor, así como un incremento de la Tensión, la Depresión y la Hostilidad. Finalmente, Chiodo et al., (2011) monitorizaron los cambios en el perfil de estado de ánimo durante un evento competitivo de jóvenes taekwondistas, destacando que entre el primer y último combate del torneo lo satlestas experimentaban aumentos significativos de las dimensiones de Hostilidad y Depresión, así como un descenso del Vigor. En su conjunto, estos resultados nos sugieren que el perfil de estado de ánimo en todos los deportistas de combate está claramente influenciado por los niveles de carga de entrenamiento regular, por las fases precompetitivas donde se llevan a cabo las rápidas bajadas de peso, por el pro- 
pio torneo de competición, y según los resultados del presente trabajo, incluso por el escaso periodo de tiempo que separa el pesaje oficial del comienzo del primer combate de un evento competitivo prioritario de la temporada.

En estas modalidades olímpicas de combate, tras el pesaje oficial, los atletas emplean el tiempo que les resta hasta el comienzo del torneo para poder recuperar, al menos en parte, los depósitos de sustrato energético y especialmente el estado de euhidratación que tenían antes de la fase de bajada de peso (Martínez-Abellán, 2013). Concretamente, los resultados de este estudio nos indican que el valor promedio de masa corporal de estos 105 deportistas de combate se incrementó un $1.5 \%(1.0 \pm 1.2 \mathrm{~kg})$ en las escasas $12-18$ horas que separaban el pesaje oficial del principio del torneo. Estos incrementos de peso son incluso menores a las descritas en estudios previos también llevados a cabo con deportistas de combate (3.5 kg - Horswill et al., 1994; 1.8 $\mathrm{kg}$ - Kordi et al., 2012), aunque en estos casos el tiempo que separó ambas mediciones fue notablemente superior (i.e., 20 h). Los resultados de ambas variables físicas y psicológicas nos indican que los competidores han podido alimentarse e hidratarse después de ser aptos en el pesaje oficial, lo que parece tener una clara relación con la mejora del estado de ánimo de los atletas, principalmente con la dimensión Vigor (Tabla 2, Figura1).

En definitiva, la información que nos puede transmitir este sencillo test es de gran ayuda para el cuerpo técnico de estas modalidades olímpicas, y sobre todo para el entrenador, que en ocasiones toma decisiones subjetivas de los estados anímicos de sus competidores, simplemente por sus apreciaciones o conductas de los deportistas. Por lo tanto, sería aconsejable que algún componente del cuerpo técnico pudiese manejar la información y sensaciones de los deportistas desde una perspectiva más objetiva, mediante este instrumento, y poder transmitirle la información al entrenador. Todos estos datos podrán ser recogidos periódicamente y trabajar los aspectos anímicos con aquellos deportistas que más lo necesiten.

Las principal limitación que presenta este trabajo es la imposibilidad de conocer la fluctuación de los estados de ánimo que acontecen en estos atletas desde una situación que pudiera considerarse estable, es decir, durante sus fases habituales de entrenamiento donde no están sometidos al estrés físico y psíquico que conllevan las fases de bajada de peso y la necesidad de acelerar la recuperación física antes del comienzo del torneo. Esta información permitiría conocer qué deportistas se ven más afectados por estas fases competitivas, y de esta forma poder programar con ellos entrenamiento psicológico específico. Futuros estudios tendrán que analizar estos cambios en los estados de ánimo durante temporadas completas de entrenamiento y competición para comprender con mayor profundidad los efectos que los diferentes estímulos tienen sobre el rendimiento psicológico de los atletas de combate. Igualmente, una posible línea de investigación futura para adentrarse más detalladamente sobre las sensaciones de los deportistas de combate, podría ser mediante el modelo tridimensional de respuesta emocional (POMS-VIC), donde se evalúa el estado de ánimo del competidor mediante tres ejes: intensidad, valencia y control (De la Vega, Ruiz, Borges, Tejero, 2014).

\section{Conclusiones}

Los principales hallazgos de este estudio nos indican que i) las 12-18 horas que separan el pesaje oficial y el comienzo del torneo en deportes de combate propician mejoras sustanciales en las dimensiones de Cólera, Fatiga y Depresión del estado de ánimo, y ii) estos cambios en el estado de ánimo no parecen estar relacionados con los niveles de fluctuación de la masa corporal que experimentan los deportistas durante este mismo periodo.

\section{Bibliografía}

1. Arruza, J. A., Arribas, S., Otaegi, O., González, O., Irazusta, S. y Ruiz, L. M. (2011). Perception of competence, mood state and stress tolerance in young high performance athletes. Anales de Psicología, 27(2), 536-543.

2. Artioli, G.G., Iglesias, R.T., Franchini, E., Gualano, B., Kashiwagura, D.B., Solis, M. Y., Benatti, F.B., Fuchs, M. y Lancha Junior, A.H. (2010). Rapid weight loss followed by recovery time does not affect judo-related performance. Journal of Sport Science, 28(1), 21-32.

3. Burke, L.M. y Cox, G.R. (2009). Nutrition in combat sports. In Combat Sports Medicine, 1-20.

4. Chiodo, S., Tessitore, A., Cortis, C., Cibelli, G., Lupo, C., Ammendolia, A., De Rosas, M. \& Capranica, L. (2011). Stress-related hormonal and psychological changes to official youth Taekwondo competitions. Scandinavian Journal of Medicine \& Science in Sports, 21(1), 111-119.

5. Degoutte, F., Jouanel, P., Begue, R.J., Colombier, M., Lac, G., Pequignot, J.M. y Filaire, E. (2006). Food restriction, performance, biochemical, psychological, and endocrine changes in judo athletes. International Journal of Sports Medicine, 27, 9-18.
6. De la Vega R., Ruiz R., Borges P.J. y Tejero C.M. (2014). Una nueva medida tridimensional del estado de ánimo deportivo: el POMS-VIC. Cuadernos de Psicología del Deporte, 14(2), 37-46.

7. De la Vega, R., Ruiz, R., Tejero, C. y Rivera, M. (2014). Relación entre estados de ánimo y rendimiento en voleibol masculino de alto nivel. Revista de Psicología del Deporte, 23(1), 49-56.

8. Filaire, E., Maso, F., Degoutte, F., Jouanel, P. y Lac G. (2001). Food restriction, performance, psychological state and lipid values in judo athletes. International Journal of Sports Medicine, 22, 454-459.

9. Franchini, E., C.J. Brito y G.G. Artioli (2012) Weight loss in combat sports: physiological, psychological and performance effects. Journal of the International Society of Sports Nutrition, 9, 52.

10. Fuentes, I., Balaguer, I., Meliá, J. L. y García-Merita, M. L. (1995). Forma abreviada del Perfil de los Estados de Ánimo (POMS). Actas del $V$ Congreso Nacional de Psicología de la Actividad Fisica y el Deporte, (pp. 29-39). Valencia: Universitat de València.

11. García-Pallarés, J., López-Gullón, J. M., Muriel, X., Díaz, A., y Iz- 
quierdo, M. (2011). Physical fitness factors to predict male Olympic wrestling performance. European Journal of Applied Physiology, 111(8), 1747-1758.

12. García Pallarés, J., López-Gullón, J. M., Torres-Bonete, M. D., y Izquierdo, M. (2012). Physical fitness factors to predict female Olympic wrestling performance and sex differences. The Journal of Strength $y$ Conditioning Research, 26(3), 794-803.

13. Hernández, R., Torres-Luque, G., \& Olmedilla, A. (2009). Relations among training volume, body weight, and profile of mood states for elite judoka during a competitive period. Perceptual and Motor Skills, 109(3), 870-880.

14. Hoffman, J. R., Bar-Eli, M. y Tenenbaum, G. (1999). An examination of mood changes and performance in a professional basketball team. Journal of Sports Medicine and Physical Fitness, 39, 74-79.

15. Horswill, C.A. (1992). Applied physiology of amateur wrestling. Sports Medicine, 14, 114-143.

16. Horswill, C. A., Scott, J. R. y Galea, P. (1989). Comparison of maximum aerobic power, maximum anaerobic power, and skinfold thickness of elite and nonelite junior wrestlers. International Journal of Sports Medicine, 10(3), 165-168.

17. Horswill C. A., Scott, J. R., Dick R., W. y Hayes, J. (1994). Influence of rapid weight gain after the weigh-in on success in collegiate wrestlers. Medicine and Science in Sports and Exercise,26(10), 1290-1294.

18. Houston, M.E., Marin, D.A., Green, H.J. y Thomson, J.A. (1981). The effect of rapid weight loss on physiological function in wrestlers. Physician and Sportsmedicine, 9(11), 73-78.

19. Kordi, R., Nourian, R., Rostami, M. y Wallance, W.A. (2012). Percentage of body fat and weight gain in participants in the tehran high school wrestling championship. Asian Journal of Sports Medicine, 3(2), 119-25.

20. Langan-Evans, C., Close, G.L. y Morton, J.P. (2011). Making Weight in Combat Sports. Strength and Conditioning Journal, 33, 25-39.

21. Lazarus, R. S. (2000). How emotions influence performance in competitive sport. The Sport Psychologist, 229-252.

22. Macedo, I. O., Sinim, M. A. y Noce, F. (2007). Utiliçaçao do POMS do monitoramento dos estados de humor antes de competiçao nas di- ferentes provas do atletismo. Cuadernos de Psicología del Deporte, 7, 1, $85-96$.

23. Marttinen, R. H., Judelson, D. A., Wiersma, L. D., y Coburn, J. W. (2011). Effects of self-selected mass loss on performance and mood in collegiate wrestlers. The Journal of Strength y Conditioning Research, 25(4), 1010-1015.

24. Martínez-Abellán A. (2013). Efectos de las pérdidas de peso y la deshidratación en deportes de combate: una revisión. Revista Euroamericana de Ciencias del Deporte, 3:59-68.

25. McNair, D. M., Lorr, M. y Droppleman, L. F. (1971). Manual for the Profile of Mood States. San Diego, CA: Educational and Industrial Testing Service.

26. Morgan, W. P. y Johnson, R. W. (1978). Personality characteristics of successful and unsuccessful oarsmen. International Journal of Sport Psychology, 9, 119-133.

27. Morgan, W. P. y Johnson, R. W. (1977). Psychologic characterization of the elite wrestler: A mental health model. Medicine and Science in Sports, 9, 55-56.

28. Morgan, W. P. y Pollock, M. L. (1977). Psychologic characterization of the elite distance runner. Annals of the New York Academy of Science, 301, 382-403.

29. Ruiz, M.C. y Hanin, Y.L. (2004). Athlete's self-perceptions of optimal states in Karate: an application of the IZOF model. Revista de Psicologia del Deporte, 13, (2), 229-244.

30. Smith, M.S. (1998). Sport specific ergometry and the physiological demands of amateur boxing. Thesis, University College, Chichester.

31. Steen, S.N. y Brownell, K.D. (1990). Patterns of weight loss and regain in wrestlers: has the tradition changed? Medicine and Science in Sports and Exercise, 22(6), 762-768.

32. Torres-Luque, G., Hernández, R., Olmedilla, A., Ortega, E. y Garatachea, N. (2013). Fluctuación del Perfil de Estados de Ánimo (POMS) en un periodo competitivo en judokas de élite. Revista de Psicología del Deporte, 22(2), 313-320.

33. Umeda, T., Nakaji, S., Shimoyama, T., Yamamoto, Y., Totsuka, M. y Sugawara, K (2004). Adverse effects of energy restriction on myogenic enzymes in judoists. Journal of Sports Sciences 22, 329-338. 